ARTICLE

https://doi.org/10.1038/s41467-019-08661-9

\title{
Size-dependent stability of ultra-small $\alpha-/ \beta$-phase tin nanocrystals synthesized by microplasma
}

\author{
Atta UI Haq (10 1, Sadegh Askari², Anna McLister ${ }^{1}$, Sean Rawlinson ${ }^{1}$, James Davis ${ }^{1}$, Supriya Chakrabarti ${ }^{1,3}$, \\ Vladimir Svrcek ${ }^{4}$, Paul Maguire (1) ${ }^{1}$, Pagona Papakonstantinou ${ }^{1} \&$ Davide Mariotti (i) $^{1}$
}

Nanocrystals sometimes adopt unusual crystal structure configurations in order to maintain structural stability with increasingly large surface-to-volume ratios. The understanding of these transformations is of great scientific interest and represents an opportunity to achieve beneficial materials properties resulting from different crystal arrangements. Here, the phase transformation from $\alpha$ to $\beta$ phases of tin ( $\mathrm{Sn}$ ) nanocrystals is investigated in nanocrystals with diameters ranging from 6.1 to $1.6 \mathrm{~nm}$. Ultra-small $\mathrm{Sn}$ nanocrystals are achieved through our highly non-equilibrium plasma process operated at atmospheric pressures. Larger nanocrystals adopt the $\beta$-Sn tetragonal structure, while smaller nanocrystals show stability with the $\alpha$-Sn diamond cubic structure. Synthesis at other conditions produce nanocrystals with mean diameters within the range $2-3 \mathrm{~nm}$, which exhibit mixed phases. This work represents an important contribution to understand structural stability at the nanoscale and the possibility of achieving phases of relevance for many applications.

\footnotetext{
${ }^{1}$ Nanotechnology \& Integrated Bio-Engineering Centre (NIBEC), Ulster University, Shore Road, Newtownabbey BT37 OQB, United Kingdom. ${ }^{2}$ Institute for Experimental and Applied Physics, Christian-Albrechts-Universität zu Kiel, Leibnizstraße 17, 24118 Kiel, Germany. ${ }^{3}$ Centre for Carbon Materials, International Advanced Research Centre for Powder Metallurgy and New Materials (ARCI), Balapur P.O., Hyderabad 500005, India. ${ }^{4}$ National Institute of Advanced Industrial Science and Technology-AIST, Central 2, Tsukuba 305-8568, Japan. Correspondence and requests for materials should be addressed to A.U.H. (email: au.haq@ulster.ac.uk) or to D.M. (email: d.mariotti@ulster.ac.uk)
} 
T in $(\mathrm{Sn})$ represents an exciting element, offering a versatile material platform to tune a range of properties. The key features of Sn are drastically affected by the crystal configuration; tin tetragonal phase ( $\beta$-Sn) in the bulk form is known to be stable at room temperature and it transforms to a diamond cubic phase $\left(\alpha\right.$-Sn) only below $\sim 13^{\circ} \mathrm{C}^{1}$, accompanied by a large increase in volume ${ }^{2}$. In order to access the properties of $\alpha$-Sn at higher temperature, a range of strategies have been investigated. In particular, the transformation temperature between $\alpha$-Sn and $\beta$-Sn can be increased with the incorporation of impurity. For instance, a very low concentration $(\sim 0.6 \mathrm{wt} \%)$ of $\mathrm{Si}$ in $\mathrm{Sn}$ shifts the transformation temperature from $\sim 13$ to $\sim 90^{\circ} \mathrm{C}^{3}$. A major difference between the two phases of $\mathrm{Sn}$ is also observed in the electronic structure as the metallic behavior of tetragonal tin becomes semi-metallic when it transforms to $\alpha$-Sn (a zerobandgap semi-metal) ${ }^{2}$. This results in drastically different optoelectronic properties.

These characteristics are again largely affected at the nanoscale as properties now depend on size and shape as much as they depend on the traditional parameters of structure and composition ${ }^{4}$. This may complicate the analysis and amplify the experimental parameter space but it also offers an opportunity for manipulating and tailoring phase transformations and corresponding properties. While there is clear experimental evidence that phase transformation of $\mathrm{Sn}$ nanocrystals (NCs) is different from bulk, the phase stability and transformation temperatures are essentially unknown for NCs of differing sizes and surface characteristics (e.g. free-standing or embedded). One of the few experimental efforts in this direction has for instance shown that $\alpha$-Sn NCs with sizes $>8 \mathrm{~nm}$ are no longer energetically stable inside the embedded Si matrix and they transform into $\beta$-Sn NCs ${ }^{4}$.

Energy storage applications have provided most of the thrust in studying Sn NCs, where alloying (e.g. with $\mathrm{Li}$ or $\mathrm{Mg}^{4-13}$ ) has been a key aspect to the research. Sn NCs are a promising candidate as an anode material for next generation batteries due to their high theoretical capacity $\left(991 \mathrm{mAhg}^{-1}\right)$ and low toxicity ${ }^{14-16}$. Also, $\mathrm{Sn}$ is more sustainable and environmentally friendly than carbon for Li-ion, Mg-ion and for the emerging $\mathrm{Na}$-ion batteries ${ }^{11,17,18}$. With a theoretical capacity three times higher than graphite ${ }^{16}$, Sn has also been used along with other technological important materials for enhancing their supercapacitive performance ${ }^{13,19,20}$.

Another major aspect that may result from nanoscale effects is quantum confinement ${ }^{21-24}$. Diamond cubic $S n(\alpha-S n)$ is a semi-metal with a low electron effective mass and excitonic confinement effects should be manifested even for relatively large $\mathrm{NCs}^{25}$; quantum confinement is therefore expected as the NCs size becomes much lower than the Bohr exciton radius, which is estimated to be around $12.56 \mathrm{~nm}$ (Supplementary Table 1). Such large value for the Bohr radius (e.g. compared to $4.5 \mathrm{~nm}$ for $\mathrm{Si}$ ) makes $\alpha$-Sn more sensitive to quantum confinement effects and any phase transformation would result in a step-change for properties deriving from the electronic structure. A first outcome of quantum confinement is the widening of the bandgap and the possibility of tuning its value from 0 up to $1.24 \mathrm{eV}^{26}$ or more by varying the NC size. Furthermore, the properties of Sn are particularly conducive to enhancing carrier multiplication, a phenomenon that is generally negligible in bulk materials and relatively limited in $\mathrm{NCs}$ with various compositions (e.g. $\mathrm{Si}, \mathrm{PbS}$, $\mathrm{PbSe})^{26}$. Opto-electronic properties in quantum confinement regime are particularly attractive for various applications $s^{5,9,27-30}$. For instance, $\alpha$-Sn NCs would enable the achievement of a groupIV direct bandgap semiconductor with a high degree of tunability for solar cell applications or for monolithic integration of optically active materials with silicon ${ }^{25,26}$.
From a fundamental point of view, there has been consistent interest in Sn NCs as demonstrated by a large number of theoretical studies (e.g., ref. ${ }^{2,11,26,29,31}$ ). The experimental efforts are however limited to relatively large NCs $(>17 \mathrm{~nm})^{10}$ or to Sn NCs embedded within bulk matrix (e.g., ref. $4,25,32,33$ ) or stabilized by using chelating agents ${ }^{34}$ and dendrimer-encapsulation meth$\mathrm{ods}^{35}$. As a consequence, many of the simulation results remain unverified as these refer to unconstrained or free-standing NCs with diameters below $5 \mathrm{~nm}$, where free-standing refers to NCs that are not embedded within a larger solid matrix. For instance, Hörmann et al. used density functional theory and argued the stability of only $\beta$-Sn NCs below $8 \mathrm{~nm}^{31}$; however, these results were in contradiction to previous experimental findings ${ }^{4,10}$.

In our work, we study the synthesis and properties of Sn NCs with diameters from $\sim 6.1 \mathrm{~nm}$ down to $1.6 \mathrm{~nm}$. We therefore report the synthesis of ultra-small $(<3 \mathrm{~nm}$ diameter) surfactantfree and free-standing Sn NCs, which is unprecedented to the best of our knowledge. We also demonstrate that $\alpha$-Sn can be stabilized at room temperature in the form of free-standing NCs with no need of a supporting matrix, which is also unprecedented to the best of our knowledge. For this purpose, we use a microplasma synthesis process that has demonstrated the required versatility to produce a range of ultra-small nanoparticles or $\mathrm{NCs}^{36-49}$. Microplasmas allow for the use of a wide range of precursors that can be supplied as gases, liquids or solids leading to an impressive compositional and structural NC tunability. Furthermore, we clarify the impact of quantum confinement, through bandgap widening, and nanoscale effects on the phase stability of Sn NCs and we show that room temperature stability of $\beta$-Sn is confirmed for NCs with diameter larger than $\sim 3 \mathrm{~nm}$. We note that at some of the synthesis conditions, Sn NCs with mixed phases are produced, which exhibit mean diameters in the range $2-3 \mathrm{~nm}$. We finally show that the Sn NCs with either $\alpha$-Sn or $\beta$-Sn phase have exceptional electrochemical capacity, which is beneficial for supercapacitors in micro-power systems, i.e., microsupercapacitors $^{50-55}$.

\section{Results}

Microplasma synthesis and characterization of tin nanocrystals. In order to achieve accurate control in the synthesis of Sn NCs, we have used a low-cost process based on an atmospheric pressure microplasma set-up (Fig. 1). Atmospheric-pressure microplasmas offer a promising platform for the synthesis of nanomaterials due to their versatile features ${ }^{36,37}$. Such microplasmas at atmospheric pressures are highly non-equilibrium in nature $^{36,42}$, which are beneficial for synthesizing highly challenging nanomaterials contrary to conventional synthesis approaches. For instance, the synthesis of silicon and silicon-carbide NCs with dimensions less than $2.5 \mathrm{~nm}$ have been successfully presented in our recent papers ${ }^{43,44}$. Furthermore, the synthesis of a variety of NCs under different configurations of microplasmas have also been demonstrated along with tuning their sizes, structure and surface functionalities ${ }^{43,45-47}$.

Our plasma reactor consists of a quartz capillary with two ring electrodes held fixed by a Perspex frame; a tin wire, used as solid precursor, is inserted in the capillary (Fig. 1a). One of the two electrodes is grounded while the other is powered with radio frequency (RF) to sustain the plasma (Fig. 1b). The inset image in Fig. 1b shows a magnified photograph where the $\mathrm{Sn}$ wire is clearly visible following a couple of hours of operation. Helium gas is used for the synthesis and Sn NCs were then collected directly in ethanol or directly deposited on carbon electrodes.

$\mathrm{Sn}$ NCs were synthesized at different $\mathrm{He}$ gas flow rates $(0.25 \mathrm{sLm}, 0.5 \mathrm{sLm}, 0.75 \mathrm{sLm}$ and $1 \mathrm{sLm})$ and collected directly in ethanol; the corresponding transmission electron microscopy 

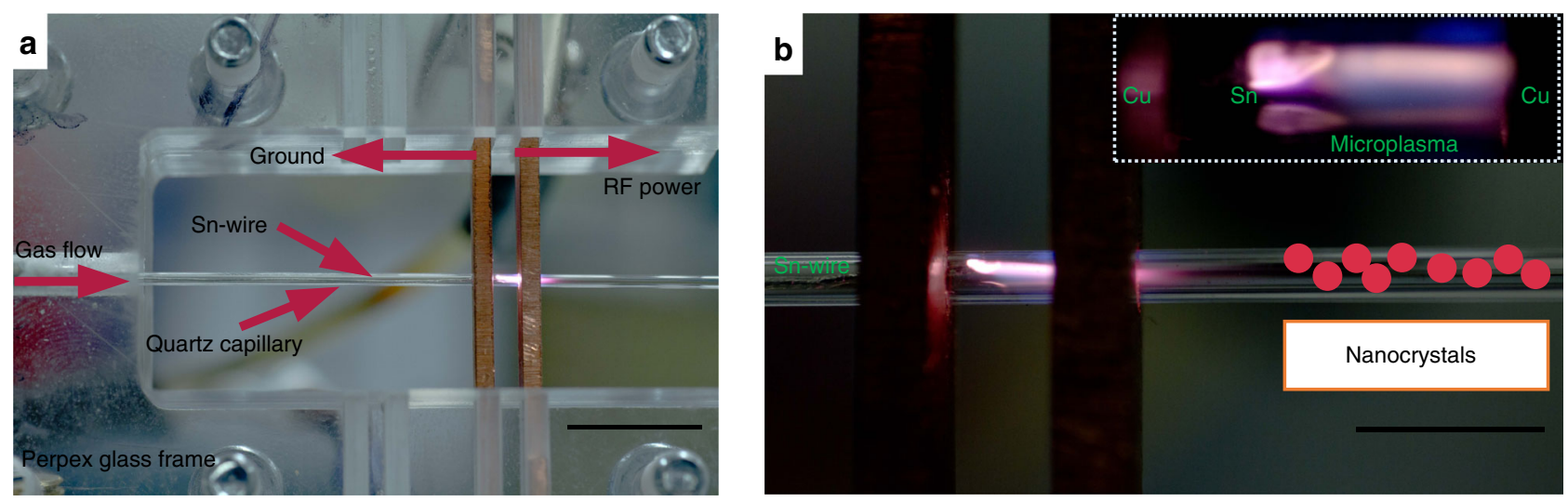

Fig. 1 Microplasma reactor configuration at atmospheric pressure. a A photographic illustration of the plasma reactor set up. A tin wire (0.25 mm diameter) is placed inside the capillary such that one end of the wire is connected to ground and the other end is suspended at the middle position between the two copper electrodes; b A high-resolution photograph of the plasma region where the Sn-wire is clearly seen (also shown in the inset image). The scale bars in (a) and (b) are 5 and $4 \mathrm{~mm}$ respectively
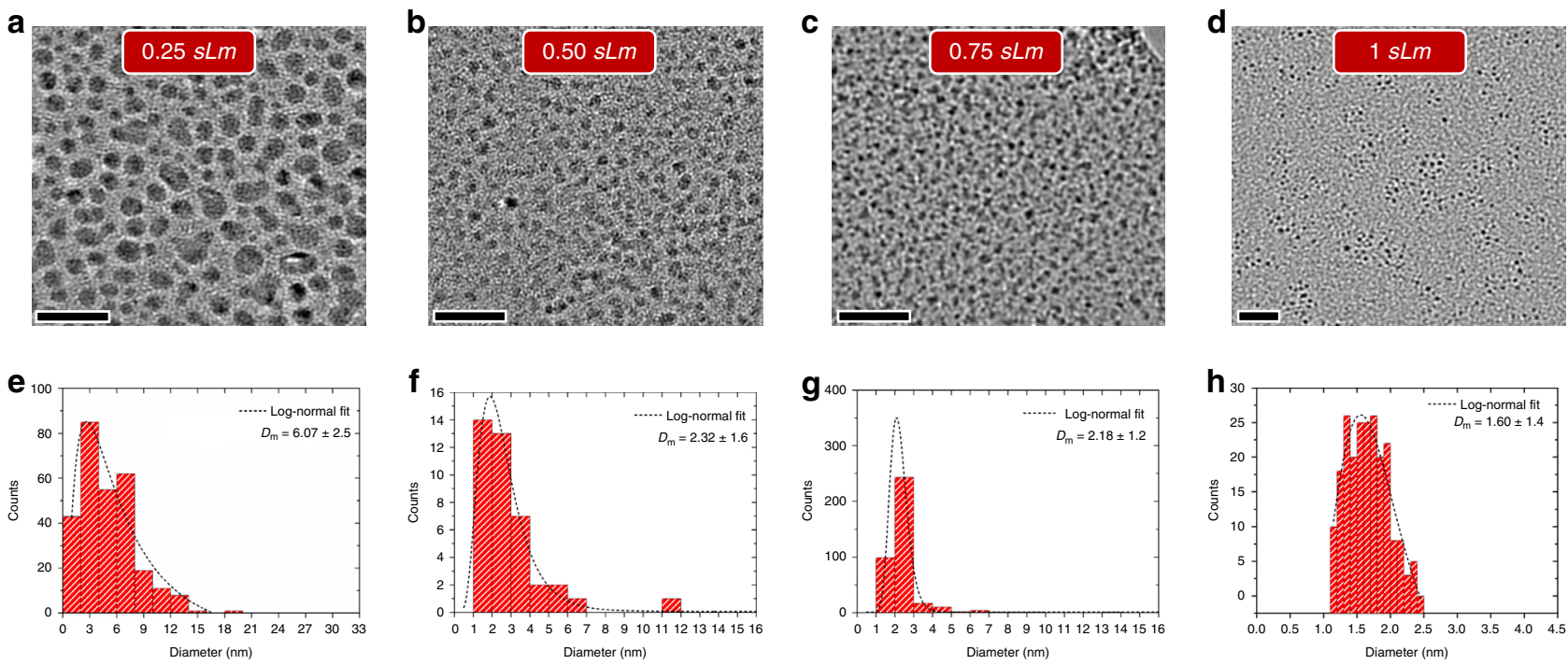

Fig. 2 Morphology of tin nanocrystals. a-d Transmission electron micrographs of Sn nanocrystals (NCs) prepared at $0.25 \mathrm{sLm}, 0.5 \mathrm{sLm}, 0.75 \mathrm{sLm}$ and $1.0 \mathrm{sLm}$ respectively; (e-h) bar charts showing size distribution of the NCs corresponding to the same micrographs in (a-d) with the average size of 6.07, 2.32, 2.18 and $1.60 \mathrm{~nm}$ respectively. The dashed curve represents the log-normal fit to the distribution. The scale bars in (a-d) is equal to $20 \mathrm{~nm}$ and the sLm corresponds to standard liter per minute

(TEM) micrographs are shown in Fig. 2a-d. The NCs were found to be well separated for each of the gas flow conditions in the plasma. The diameter of the NCs decreases with increasing gas flow rate due to the lower residence time of the particles in the plasma (see discussion on the formation mechanisms further below). The shape of the NCs has also been affected by the gas flow rates. The NCs prepared at higher gas flow rates (e.g., $1 \mathrm{sLm}$ ) showed highly spherical shapes (e.g., Fig. 2d) while deviation from their spherical nature was observed at lower gas flow rates (e.g., Fig. 2a). The NCs at $0.25 \mathrm{sLm}$, where plasma heating effect is higher, were found to be slightly elongated in their shape as can be seen in Fig. 2a.

Figure $2 \mathrm{e}-\mathrm{h}$ shows bar chart diagram representing the size distributions in each of the gas flow conditions. The average size of the NCs at $0.25 \mathrm{sLm}, 0.5 \mathrm{sLm}, 0.75 \mathrm{sLm}$ and $1.0 \mathrm{sLm}$ was determined by fitting log-normal distributions, which produced mean values of $6.07,2.32,2.18$ and $1.60 \mathrm{~nm}$, respectively. The size of Sn NCs increases by decreasing the flow of helium gas as shown in Fig. 2 (also summarized in Supplementary Fig. 1).
Figure 3 shows representative TEM images and selected area electron diffraction (SAED) patterns that support the existence of different phases for different synthesis conditions (see Supplementary Table 2, additional images and corresponding fast Fourier transforms in Supplementary Figs 2-9). For NCs with average diameter of $6.07 \mathrm{~nm}$, the lattice fringes measured 0.20 and $0.16 \mathrm{~nm}$, which correspond to the (211) and (301) planes of $\beta$-Sn, respectively (Fig. 3a). The tetragonal structure was further confirmed by SAED patterns matching with the $\beta$ Sn (JCPDS: 01-089-4898) as shown in Fig. 3e. A mixture of phases of $\alpha$-Sn and $\beta$-Sn was found for the NCs with average diameters of 2.32 and $2.18 \mathrm{~nm}$ (Fig. 3b, c). The lattice fringes of NCs with $2.32 \mathrm{~nm}$ average diameter were 0.18 and $0.29 \mathrm{~nm}$ that represents (222) and (200) planes of $\alpha$-Sn and $\beta$-Sn respectively as shown in Fig. 3b. The corresponding diffraction rings also matched with the crystallographic data for Sn (JCPDS/ICSD taken from Diffracplus PDFMAINT: $\beta$-Sn $=01-089-4898 /$ 076149; $\alpha$-Sn $=01-089-4789 / 076040)$ which can be seen in Fig. 3f. Similarly, for NCs with average diameter of $2.18 \mathrm{~nm}$, 

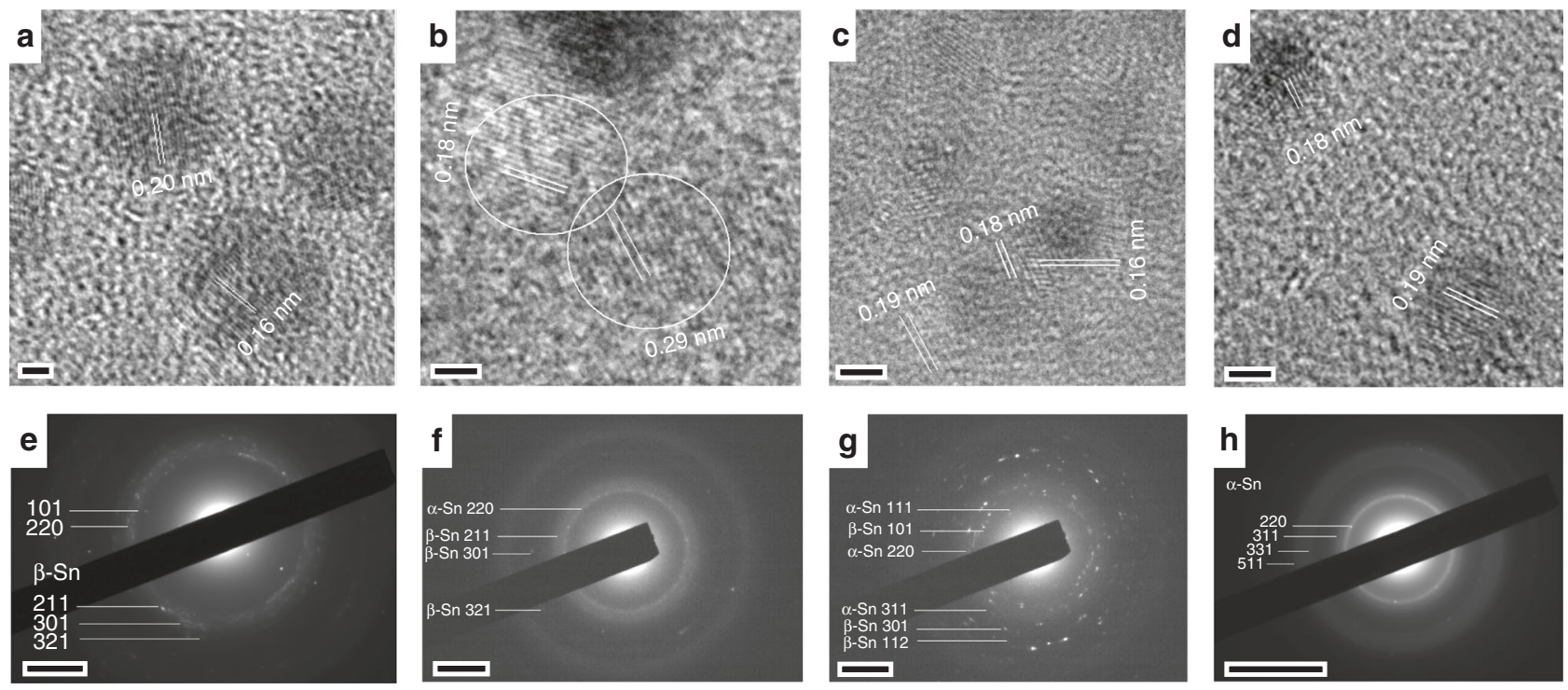

Fig. 3 Crystal structural transformations in tin nanocrystals. a-d Representative high-resolution transmission electron micrographs corresponding to Sn nanocrystals (NCs) prepared at $0.25 \mathrm{sLm}, 0.5 \mathrm{sLm}, 0.75 \mathrm{sLm}$ and $1.0 \mathrm{sLm}$ and with corresponding average diameter of $6.07,2.32,2.18$ and $1.60 \mathrm{~nm}$;

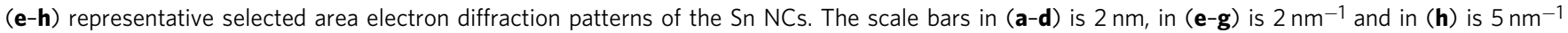

lattice fringes corresponding to the planes in both $\alpha$-Sn and $\beta$-Sn were observed, i.e. 0.18 and $0.19 \mathrm{~nm}$ corresponding to (222) and (311) planes in $\alpha$-Sn and $0.16 \mathrm{~nm}$ from the (301) plane in $\beta$-Sn (Fig. 3c). The respective diffraction pattern is also shown in Fig. $3 \mathrm{~g}$ that confirms the rings with randomly oriented crystals of mixed phases of Sn. Fig. 3d, h confirms the stability of $\alpha-\mathrm{Sn}$ at room temperature for the smallest NCs $(\sim 1.60 \mathrm{~nm})$ as the lattice fringes and diffraction rings matches with the standard data charts. Lattice fringes corresponding to the planes in $\alpha$-Sn were observed, i.e. 0.18 and $0.19 \mathrm{~nm}$ corresponding to (222) and (311) planes (Fig. 3d). The respective diffraction pattern is also shown in Fig. $3 \mathrm{~h}$ that confirms the rings belonging to $\alpha$-Sn.

Therefore, with the NCs diameter reduction from 6.07 to $1.60 \mathrm{~nm}$, different phases were observed, i.e. $\beta$-Sn for the larger NCs, mix phases for intermediate sizes and $\alpha$-Sn for the smallest NCs. The different phases were confirmed by both the measurement of lattice fringes applied to a number of single NCs as well as by analyzing SAED patterns which depict the crystal structure of an ensemble of NCs.

In the microplasma synthesis process, Sn atoms are produced from the heated wire ${ }^{56,57}$, which are subsequently ionized. Neutral and ionized Sn-atoms contribute to the initial phases of nucleation and then to the NCs growth. The gas flow has a direct impact on the overall background gas temperature (higher temperature for lower flow rates) where Sn-atom production rates at the wire are higher for higher temperatures. Therefore, the higher density of $\mathrm{Sn}$ atoms/ions produced at the wire together with a longer residence time are the reasons for larger NCs at lower gas flow rates. The opposite occurs for higher gas flow rates, with a lower $\mathrm{Sn}$ atoms/ions density and shorter residence time leading to smaller NCs.

As demonstrated in the TEM analysis, the larger NCs are preferably stabilized with the tetragonal phase while the diamond cubic phase is preferred at smaller sizes. It has been reported that the stabilization of thermodynamically unstable structures can be achieved by embedding them in matrix, by growing them on lattice-matched substrates and by doping or growing a shell around them $4,9,10,25,33$. In our process, at higher gas flow rates, nucleation and growth is fast whereby energy is continuously supplied to the growing NCs through frequent collisions in the plasma with a range of energetic species; this is then followed by fast quenching as the NCs are collected outside the plasma. These are common phenomena in plasma processes ${ }^{58,59}$ which contribute to maintaining the diamond cubic structure $(\alpha-\mathrm{Sn})$ and the crystalline nature; the conditions of these plasma processes also depart from the conditions applied in simulations which predict $\beta$-Sn NCs to be always more stable ${ }^{2}$. Theoretical simulations have shown that NCs with diameters in the range 1-2 nm generally tend to relax in an amorphous phase ${ }^{2}$, while our $1.6 \mathrm{~nm}$ NCs confirm the contribution of plasma-induced NC selective heating and crystallization ${ }^{46}$. Lower gas flow rates, when higher background temperatures are expected, allow for relaxation, leading to the stabilization in the tetragonal structure. Therefore the microplasma process offers a range of unique features that have facilitated the synthesis of these NCs and which are not available to conventional techniques. The precursor supply at high pressure allows for fast aggregation kinetics instead of slow surface growth, the former resulting in ultra-small NCs while the latter generally leads to larger NCs. Selective NC heating and charging are plasma-specific effects and in this case have allowed NCs to reach the necessary crystallization temperature while remaining separate from each other. Fast quenching is another unique feature of microplasmas, which has contributed to the instantaneous stabilization of the respective NCs phases.

The mixed phases observed for the NCs with diameters in the range of $2-3 \mathrm{~nm}$ indicate a transition region in the synthesis parameter space. This could originate from NCs being subjected to different environments due to the strong gradients (temperatures, species densities etc.) present within the plasma volume or due to small process conditions drifts over time. The observation of mixed phases only at given synthesis conditions may suggest that some set-up improvements could extend the synthetic capabilities; at the same time it also highlights the sophistication of this process, which has shown the possibility of stabilizing of $\alpha-/ \beta$-Sn separately at other synthesis conditions. The exploration of a larger parameter space and possibly by de-coupling the precursor supply from the nucleation/growth steps may allow extending the diameter range where either $\alpha$ - or $\beta$-Sn can be stabilized. The use of liquid/gaseous precursors and the growth of 
stabilizing shells are also avenues that could be investigated further.

Quantum confinement in tin nanocrystals. The $\alpha$-phase stabilized in our NCs (1.6 nm mean diameter) is expected to exhibit direct semiconducting behavior (vs. metallic of the $\beta$-phase) with strong quantum confinement (diameter $<12.56 \mathrm{~nm}$ Bohr radius). We have therefore carried out measurements to determine the bandgap of the smallest $\alpha$-phase NCs and to verify the opening of the bandgap as expected from quantum confinement. Figure $4 \mathrm{a}$ shows the bandgap determined experimentally for the first time for the $\alpha$-Sn NCs $(1.6 \mathrm{~nm})$ from the UV-Vis transmission measurements and with an integrating sphere (see also Supplementary note 1 and Supplementary Fig. 10). A bandgap of $2.2 \mathrm{eV}$ was estimated from the Tauc plot (Fig. 4a). The role of strong quantum confinement in our ultra-small semiconducting $\alpha$-Sn NCs opens the bandgap as expected also from theoretical calculations reported by Allan et al. ${ }^{26}$. In Fig. $4 \mathrm{~b}$ we report the bandgap values reported by Allan et al. ${ }^{26}$ (highlighted inside the blue rectangular region), which were calculated for diameters $>2 \mathrm{~nm}$. In order to compare with our smaller NCs we have extrapolated

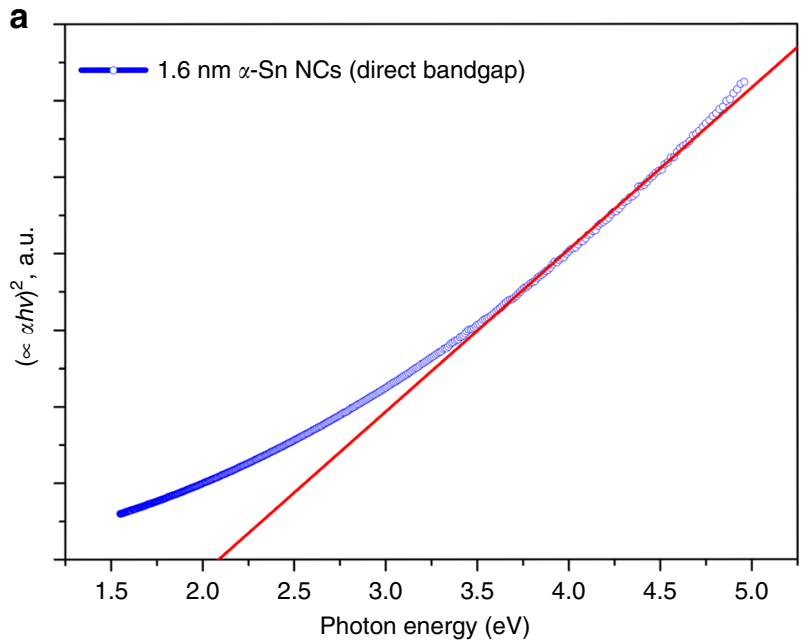

to $1.6 \mathrm{~nm}$ by fitting with an exponential curve. This shows that our experimental results are in very close agreement with the theoretical calculations reported by Allan et al. ${ }^{26}$.

Electrochemical performance of tin nanocrystals. Owing to the energetic and high surface area of our plasma-produced Sn NCs and their practical importance in many energy-related devices, we finally assess the properties of our Sn NCs for one of their potential applications. In particular, we tested the electrochemical capacitance of Sn NCs (see Supplementary Figs 11-13), which is relevant for application as an electrode material for microsupercapacitors ${ }^{60,61}$. Fig. 5a shows the cyclic voltammograms of 1.6 and $6.1 \mathrm{~nm} \mathrm{Sn} \mathrm{NCs} \mathrm{at} \mathrm{a} \mathrm{scan} \mathrm{rate} \mathrm{of} 40 \mathrm{mV} \mathrm{s}^{-1}$. The potential window range was taken from -0.2 to $0.1 \mathrm{~V}$. It can be noted that the shape of the curves show a rectangular capacitive behavior depicting fast and easy diffusion/migration of charges ${ }^{62}$; this is representative of a highly reversible non-faradaic reaction at the electrode/electrolyte interface, which is characteristic of electrical double layer capacitors ${ }^{61}$.

The areal capacitance of these NCs was estimated (see Supplementary note 2) with the active electrode area of

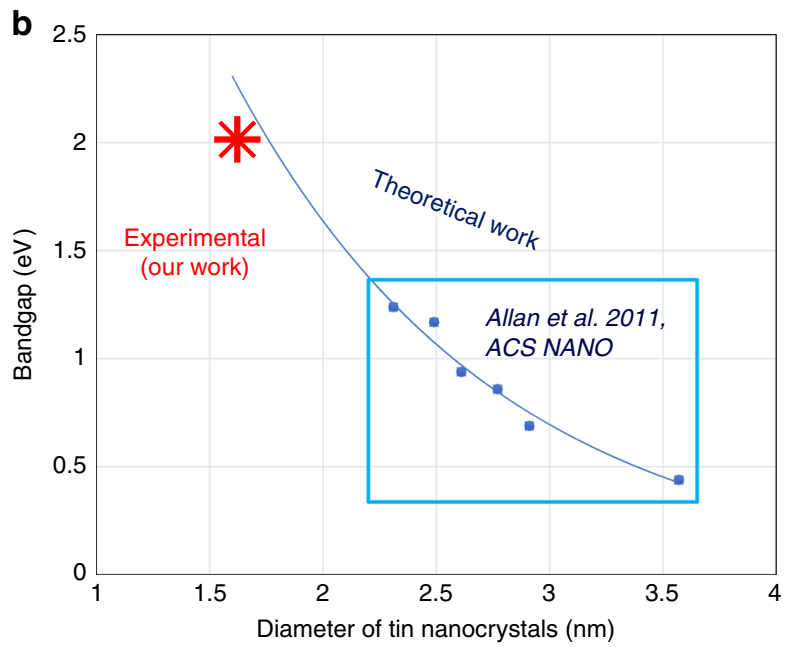

Fig. 4 Quantum confinement induced bandgap widening in tin ( $\mathrm{Sn}$ ) nanocrystals (NCs). a Tauc plot of $1.6 \mathrm{~nm}$ Sn NCs and linear fit which estimates the bandgap to be $2.2 \mathrm{eV}$; as per Tauc plot of direct semiconductors, $C^{\prime}$ is the proportionality constant, $\alpha$ is the absorption coefficient, $h \nu$ is the energy ( $h$ is Planck's constant and $\nu$ is the frequency). b Bandgap values (blue dots) for Sn NCs from theoretical calculations ${ }^{26}$ and fitted exponential curve (blue line). The red star corresponds to our experimental result
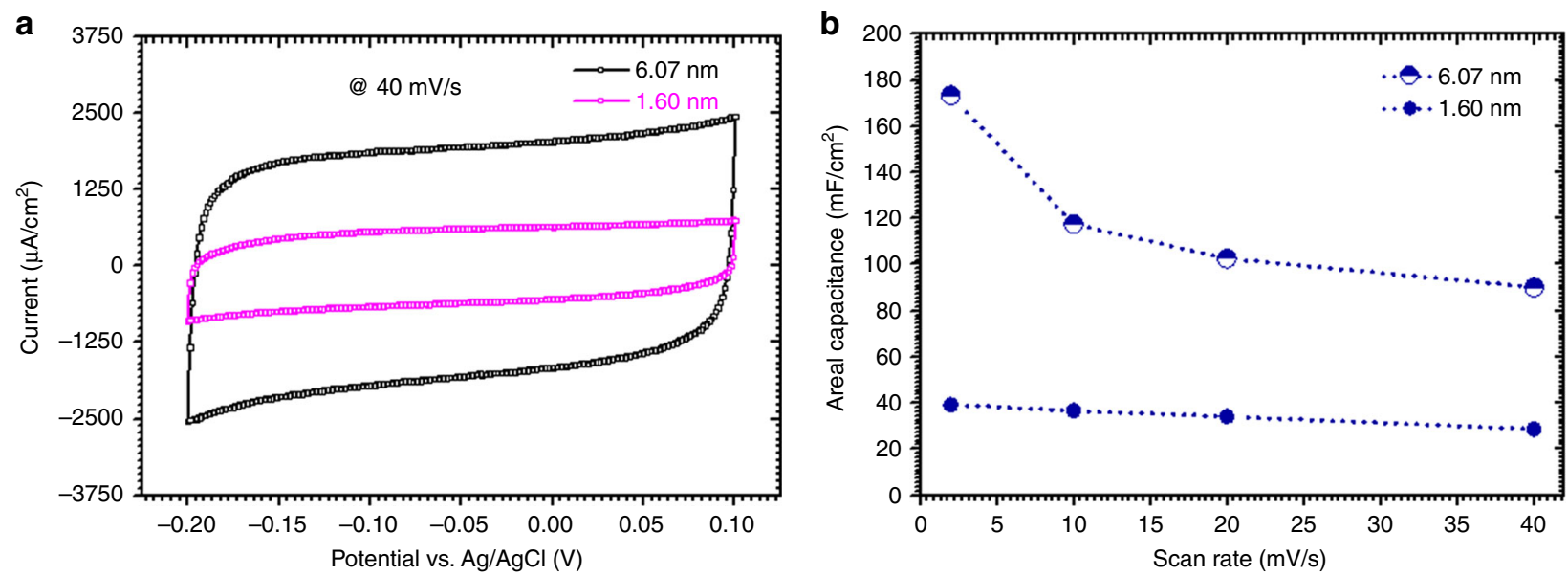

Fig. 5 Electrochemical performance of tin ( $\mathrm{Sn}$ ) nanocrystals. a Cyclic voltammograms of tin ( $\mathrm{Sn}$ ) nanocrystals ( $\mathrm{NCs}$ ) in $0.1 \mathrm{M}$ sodium hydroxide ( $\mathrm{NaOH}$ ) solution attained at scan rate $40 \mathrm{mV} \mathrm{s}^{-1}$ respectively; $\mathbf{b}$ evolution of the relative areal capacitances of Sn NCs versus scan rates 
$\sim 0.04 \mathrm{~cm}^{2}$; the areal capacitance is a more significant performance metric for micro-supercapacitors (e.g. vs. gravimetricbased measurements) as the weight of the active material is negligible and the available area is generally limited for integrating components into a system ${ }^{50,51,53,55,63}$. As can be seen in Fig. 5b, a remarkably high capacitance of $173 \mathrm{mF} \mathrm{cm}^{-2}$ and 39 $\mathrm{mF} \mathrm{cm}^{-2}$ was measured, at nearly equilibrium conditions $\left(\sim 2 \mathrm{mV} \mathrm{s}^{-1}\right)$; this is the highest areal capacitance measured for any $\mathrm{Sn}$-based electrode, to the best of our knowledge. Furthermore, our reported areal capacitance values are also much higher than the carbon electrodes (see Supplementary Fig. 14) and those of the reported values for metallic nanoparticle-based, carbonbased supercapacitors and others ${ }^{50,53-55,63-67}$. Although, ruthenium oxide $\left(\mathrm{RuO}_{2}\right)$ has been reported $53,68,69$ with higher capacitance, $\mathrm{Sn}$-based devices still present much greater benefits originating from the much lower cost and much higher availability of $\mathrm{Sn}$ vs. $\mathrm{Ru}$. It should also be noted that $\mathrm{RuO}_{2}$ devices rely on reversible faradaic surface reactions (i.e. pseudocapacitors) and therefore their scan rates are intrinsically limited ${ }^{53}$ compared to those available with electrical doublelayers.

The higher capacitance for larger NCs $(6.07 \mathrm{~nm})$ compared to that from smaller NCs $(1.60 \mathrm{~nm})$ could be due to the relatively lower intrinsic electrical resistivity of purely metallic $(\beta-\mathrm{Sn})$ compared to that of the semiconducting phase $(\alpha-\mathrm{Sn})$ that may affect the charge transport to the current collector ${ }^{10}$. The smaller NCs with a diamond cubic phase $(\alpha$-Sn) show overall excellent capacitive performance with the capacitance values still exceptionally high and nearly constant with increasing scan rates (Fig. 5b). The capacity retention in the larger $\beta$-Sn NCs is decreasing with increasing scan rate $\left(2-40 \mathrm{mV} \mathrm{s}^{-1}\right)$; this is related to insufficient time for the electrolyte to adsorb and desorb on the electrode surface and an increased resistance. This increase in the internal resistance is expected due to the poor packing of the larger NCs along with their lower surface area compared to smaller NCs as reported elsewhere ${ }^{70}$.

\section{Discussion}

In this work, size and structure selective NCs of Sn have been successfully synthesized using plasma synthesis operated at atmospheric pressure. The size and crystalline phase of the NCs was strongly dependent on the gas flow rate. NCs produced with flow rates at $0.25 \mathrm{sLm}, 0.5 \mathrm{sLm}, 0.75 \mathrm{sLm}$ and $1.0 \mathrm{sLm}$ resulted in NCs with mean diameters of $6.07,2.32,2.18$ and $1.60 \mathrm{~nm}$, respectively. The lowest flow rate, produced NCs of the largest mean diameter and of $\beta$-Sn phase; increasing the flow rate resulted in NCs with progressively smaller diameters $(2-3 \mathrm{~nm})$ and mixed $\alpha-/ \beta$-Sn phases. Finally, the higher gas flow rate resulted in much smaller NCs with regular and spherical shapes and with a diamond cubic structure of Sn. The stabilization of $\alpha$ Sn was found to be at $1.60 \mathrm{~nm}$. A phase transition was observed from diamond cubic $(\alpha-S n)$ to tetragonal structure $(\beta-S n)$ with decreasing gas flow rates. The experimental results first demonstrate the possibility of stabilizing $\alpha$-Sn in free-standing and surfactant-free NCs without any supporting matrix. The smallest NCs $(1.6 \mathrm{~nm}, \alpha-\mathrm{Sn})$ also exhibit quantum confinement and semiconducting behavior with a bandgap widening as predicted by theoretical calculations. Areal capacitances of $173 \mathrm{mF} \mathrm{cm} \mathrm{m}^{-2}$ and $39.2 \mathrm{mF} \mathrm{cm}^{-2}$ were estimated for larger $(6.07 \mathrm{~nm})$ and smaller NCs $(1.60 \mathrm{~nm})$ respectively. While further work and full device characterization will be required, the high areal capacitance of $173 \mathrm{mF} \mathrm{cm}^{-2}$ represents the best value achieved for $\mathrm{Sn}$ $\mathrm{NC}$ electrodes, to the best of our knowledge, and compares favorably with other metallic nanoparticle and carbon-based supercapacitor electrodes. The smaller NCs also exhibited retention of the areal capacitance for a wide range of scan rates. The results also show that plasma-based processes allow for exquisite synthesis capabilities leading to Sn NCs with unprecedented dimensions; in addition atmospheric pressure operation and the use of a solid precursor offer clear advantages for the deployment and direct integration in device manufacturing ${ }^{52}$ The work therefore advances the current state of the art, provides opportunities to compare and verify theoretical results and offer important directions for applications.

\section{Methods}

Details of the microplasma synthesis process. Our plasma reactor consists of a quartz capillary (100 $\mathrm{mm}$ long) with an internal and external diameter of 0.75 and $1.0 \mathrm{~mm}$, respectively (Fig. 1). Two ring electrodes with the thickness of $1 \mathrm{~mm}$ each and with a spacing of $2 \mathrm{~mm}$ are held fixed around the capillary by a Perspex frame. A tin $(\mathrm{Sn})$ wire $(0.25 \mathrm{~mm}$ diameter, Goodfellow Ltd.) is inserted in the capillary such that one end is kept fixed at $1 \mathrm{~mm}$ from the powered electrode. The Sn-wire acts as a sacrificial electrode and Sn precursor. A radio frequency (RF) power of $40 \mathrm{~W}$ (applied power) was selected as an optimized power for sustaining the plasma as shown in Fig. 1b. The flow rate of helium gas was varied from $0.25 \mathrm{sLm}$ to $1.00 \mathrm{sLm}$. The plasma process produces $2-6 \mu \mathrm{g} \mathrm{min}{ }^{-1}$ depending on synthesis conditions (Supplementary note 3 and Supplementary Table 3 ). This process has been optimized for investigating the fundamental nature of these small NCs. However scaling the process throughput will be possible through known approaches involving e.g. plasma geometry expansion in one dimension ${ }^{39,71}$ or by using multiple parallel plasma reactors arrays ${ }^{36,48,72}$. In any such development, the power delivery and flow will require enhanced feedback control to maintain the plasma conditions within the precise parameter range discovered here.

Materials Characterization. The morphology and structure of the Sn NCs were examined by TEM and SAED using a JEOL JEM-2100F electron microscope operated at $200 \mathrm{keV}$. The samples for TEM were prepared by drop casting Sn NCs/ ethanol colloids on holy carbon mesh/Cu TEM grids and then were allowed to dry. The analysis of the size distribution of the Sn NCs was carried out by processing TEM micrographs with ImageJ software. The lattice fringes were measured using Gatan Microscopy Suit Software while the analysis of SAED patterns were analyzed manually by measuring the diameters of each ring and comparing them with standard crystallographic JCPDS cards.

Ultraviolet-visible (UV-Vis) spectroscopy measurements were carried out on a PerkinElmer Lambda 650S accessorized with $150 \mathrm{~mm}$ integrating sphere. Sn NCs were deposited directly on quartz slides to form a film. The measurements were carried out in standard transmission mode first and then within the integrating sphere to account for reflection (see Supplementary note 4 for more details). The electrochemical characterization was performed in $0.1 \mathrm{M}$ sodium hydroxide using cyclic voltammetry with a conventional three electrode cell in which platinum wire and $\mathrm{Ag} / \mathrm{AgCl}(3 \mathrm{M} \mathrm{NaCl})$ half-cell served as counter and reference electrode respectively (Supplementary Fig. 11). All the electrochemical measurements were made using a Type III Micro Autolab (EcoChimie, Netherlands) potentiostat. The samples for cyclic voltammetry (CV) analysis were prepared by directly depositing $\mathrm{Sn}$ NCs on a $0.04 \mathrm{~cm}^{2}$ area of screen printed carbon electrodes.

\section{Data availability}

The data that support the findings of this study are available within the article and the Supplementary Information, and available from the corresponding author upon request

Received: 12 June 2018 Accepted: 18 January 2019

Published online: 18 February 2019

\section{References}

1. Busch, G. A. \& Kebn, R. Semiconducting properties of gray tin. Solid State Phys. 11, 1-40 (1960).

2. Sabet, S. \& Kaghazchi, P. Communication: nanosize-induced restructuring of Sn nanoparticles. J. Chem. Phys. 140, 2012-2016 (2014).

3. Gallerneault, W. M. T., Vnuk, F. \& Smith, R. W. Silicon-stabilized grey tin. J. Appl. Phys. 54, 4200 (1983).

4. Arslan, I., Yates, T. J. V., Browning, N. D. \& Midgley, P. A. Embedded nanostructures revealed in three dimensions. Science 309, 2195-2198 (2005).

5. Zhu, Z. et al. Ultrasmall Sn nanoparticles embedded in nitrogen-doped porous carbon as high-performance anode for lithium-ion batteries. Nano. Lett. 14, 153-157 (2014).

6. Wang, J. W., Liu, X. H., Mao, S. X. \& Huang, J. Y. Microstructural evolution of tin nanoparticles during in situ sodium insertion and extraction. Nano Lett. 12, 5897-5902 (2012). 
7. Parent, L. R. et al. Realizing the full potential of insertion anodes for Mg-ion batteries through the nanostructuring of Sn. Nano Lett. 15, 1177-1182 (2015).

8. Zhang, W. M. et al. Tin-nanoparticles encapsulated in elastic hollow carbon spheres for high-performance anode material in lithium-ion batteries. Adv. Mater. 20, 1160-1165 (2008).

9. Im, H. S. et al. Phase evolution of tin nanocrystals in lithium ion batteries. ACS Nano 7, 11103-11111 (2013).

10. Oehl, N. et al. Critical size for the $B$ - to $\alpha$-transformation in tin nanoparticles after lithium insertion and extraction. Cryst Eng Comm 17, 3695-3700 (2015).

11. Wang, Z. et al. Comparison of tetragonal and cubic tin as anode for $\mathrm{Mg}$ ion batteries. ACS Appl. Mater. Interfaces 6, 6786-6789 (2014).

12. Qin, J. et al. Graphene networks anchored with sn @ graphene as lithium ion battery. ACS Nano 8, 1728-1738 (2014).

13. Qin, Z., Li, Z. J., Zhang, M., Yang, B. C. \& Outlaw, R. A. Sn nanoparticles grown on graphene for enhanced electrochemical properties. J. Power Sources 217, 303-308 (2012).

14. Chen, J., Yang, L., Zhang, Z., Fang, S. \& Hirano, S. Mesoporous $\mathrm{TiO}_{2}-\mathrm{Sn} @ \mathrm{C}$ core-shell microspheres for Li-ion batteries. Chem. Commun. (Camb.). 49, 2792-2794 (2013)

15. Hou, H. et al. Facile preparation of $\mathrm{Sn}$ hollow nanospheres anodes for lithiumion batteries by galvanic replacement. Mater. Lett. 128, 408-411 (2014).

16. $\mathrm{Li}, \mathrm{X}$. Y. et al. Inserting $\mathrm{Sn}$ nanoparticles into the pores of $\mathrm{TiO}_{2-\mathrm{x}}-\mathrm{C}$ nanofibers by lithiation. Adv. Funct. Mater. 26, 376-383 (2016).

17. Slater, M. D., Kim, D., Lee, E. \& Johnson, C. S. Sodium-Ion batteries. Adv. Funct. Mater. 23, 947-958 (2013).

18. Wang, B., Luo, B., Li, X. \& Zhi, L. The dimensionality of Sn anodes in Li-ion batteries. Mater. Today 15, 544-552 (2012)

19. Zhao, Y. et al. Synthesis of $\mathrm{Sn}$-doped $\mathrm{Mn}_{3} \mathrm{O}_{4} / \mathrm{C}$ nanocomposites as supercapacitor electrodes with remarkable capacity retention. Mater. Lett. 118, 80-83 (2014).

20. Jang, Y.-S. et al. Improved supercapacitor potential and antibacterial activity of bimetallic CNFs-Sn-ZrO $\mathrm{Zr}_{2}$ nanofibers: fabrication and characterization. RSC Adv. 4, 17268 (2014).

21. Jena, P. \& Sun, Q. Super atomic clusters: design rules and potential for building blocks of materials. Chem. Rev. 118, 5755-5870 (2018).

22. Karakoti, A. S., Sanghavi, S., Nachimuthu, P., Yang, P. \& Thevuthasan, S. Probing the size- and environment-induced phase transformation in CdSe quantum dots. J. Phys. Chem. Lett. 2, 2925-2929 (2011).

23. Bera, D., Qian, L., Tseng, T.-K. \& Holloway, P. H. Quantum dots and thei multimodal applications: a review. Materials 3, 2260-2345 (2010).

24. Parak, W. J., Manna, L. \& Nann, T. Fundamental principles of quantum dots. Nanotechnology 73-96 https://doi.org/10.1002/9783527628155.nanotech004 (2010).

25. Ragan, R. \& Atwater, H. A. Diamond cubic Sn-rich nanocrystals: Synthesis, microstructure and optical properties. Appl. Phys. A Mater. Sci. Process. 80, 1335-1338 (2005).

26. Allan, G. \& Delerue, C. Optimization of carrier multiplication for more effcient solar cells: the case of Sn quantum dots. ACS Nano 5, 7318-7323 (2011).

27. Küfner, S., Furthmüller, J., Matthes, L. \& Bechstedt, F. Corrigendum: Optical absorption and emission of $\alpha-S n$ nanocrystals from first principles. Nanotechnology 24, 469501 (2013).

28. Zhou, X., Lu, J., Li, L. \& Wang, Z. Preparation of crystalline Sn-Doped $\mathrm{TiO}_{2}$ and its application in visible-light photocatalysis. J. Nanomater. 2011, 432947 (2011).

29. Jensen, R. V. S., Garm Pedersen, T. \& Pedersen, K. Optical properties and size/ shape dependence of $\alpha$-Sn nanocrystals by tight binding. Phys. Status Solid Curr. Top. Solid State Phys. 8, 1002-1005 (2011).

30. Kjeldsen, M. M., Hansen, J. L., Pedersen, T. G., Gaiduk, P. \& Larsen, A. N. Tuning the plasmon resonance of metallic tin nanocrystals in Si-based materials. Appl. Phys. A Mater. Sci. Process. 100, 31-37 (2010).

31. Hörmann, N. G., Gross, A. \& Kaghazchi, P. Semiconductor-metal transition induced by nanoscale stabilization. Phys. Chem. Chem. Phys. 17, 5569-5573 (2015).

32. Huang, S. et al. Fabrication and characterization of tin-based nanocrystals. J. Appl. Phys. 102, 114304 (2007).

33. Fyhn, M. F., Chevallier, J., Larsen, A. N., Feidenhans'l, R. \& Seibt, M. $\alpha$-Sn and $\beta$-Sn precipitates in annealed epitaxial $\mathrm{Si}_{0.95} \mathrm{Sn}_{0.05}$. Phys. Rev. B 60, 5770-5777 (1999).

34. Wang, Y., Lee, J. Y. \& Deivaraj, T. C. Tin nanoparticle loaded graphite anodes for li-ion battery applications. J. Electrochem. Soc. 151, A1804 (2004).

35. Bhandari, R. et al. Electrochemical activity of dendrimer-stabilized tin nanoparticles for lithium alloying reactions. Langmuir 31, 6570-6576 (2015).

36. Mariotti, D. \& Sankaran, R. M. Microplasmas for nanomaterials synthesis. J. Phys. D Appl. Phys. 43, 323001 (2010).

37. Chiang, W.-H. \& Mohan Sankaran, R. Linking catalyst composition to chirality distributions of as-grown single-walled carbon nanotubes by tuning $\mathrm{Ni}_{\mathrm{x}} \mathrm{Fe}_{1-\mathrm{x}}$ nanoparticles. Nat. Mater. 8, 882-886 (2009).
38. Lin, L. \& Wang, Q. Microplasma: a new generation of technology for functional nanomaterial synthesis. Plasma Chem. Plasma Process. 35, 925-962 (2015).

39. Wagner, A. J., Mariotti, D., Yurchenko, K. J. \& Das, T. K. Experimental study of a planar atmospheric-pressure plasma operating in the microplasma regime. Phys. Rev. E 80, 065401 (2009).

40. McKenna, J. et al. Synthesis and surface engineering of nanomaterials by atmospheric-pressure microplasmas. Eur. Phys. J. Appl. Phys. 56, 24020 (2011).

41. Burakov, V. S. et al. Atmospheric pressure plasma in contact with liquid and its application for nanoparticles synthesis. Eur. Phys. J. Appl. Phys. 79, 10801 (2017).

42. Mariotti, D. Nonequilibrium and effect of gas mixtures in an atmospheric microplasma. Appl. Phys. Lett. 92, 151503-151506 (2008).

43. Askari, S. et al. Ultra-small photoluminescent silicon-carbide nanocrystals by atmospheric-pressure plasmas. Nanoscale 8, 17141-17149 (2016).

44. Macias-Montero, M. et al. Energy band diagram of device-grade silicon nanocrystals. Nanoscale 8, 6623-6628 (2016).

45. Maguire, P. et al. Continuous in-flight synthesis for on-demand delivery of ligand-free colloidal gold nanoparticles. Nano Lett. 17, 1336-1343 (2017).

46. Askari, S., Levchenko, I., Ostrikov, K., Maguire, P. \& Mariotti, D. Crystalline Si nanoparticles below crystallization threshold: Effects of collisional heating in non-thermal atmospheric-pressure microplasmas. Appl. Phys. Lett. 104, 163103 (2014)

47. Mariotti, D., Vladimir Švrček, V., Hamilton, J. W. J., Schmidt, M. \& Kondo, M. Silicon nanocrystals in liquid media: Optical properties and surface stabilization by microplasma-induced non-equilibrium liquid chemistry. $A d v$ Funct. Mater. 22, (954-964 (2012).

48. Mariotti, D. et al. Low-temperature atmospheric pressure plasma processes for 'green' third generation photovoltaics. Plasma Process. Polym. 13, 70-90 (2016).

49. Kortshagen, U. R. et al. Nonthermal plasma synthesis of nanocrystals: Fundamental principles, materials, and applications. Chem. Rev. 116, 11061-11127 (2016).

50. Liu, W.-W., Feng, Y.-Q., Yan, X.-B., Chen, J.-T. \& Xue, Q.-J. Superior microsupercapacitors based on graphene quantum dots. Adv. Funct. Mater. 23, 4111-4122 (2013).

51. Gogotsi, Y. \& Simon, P. True performance metrics in electrochemical energy storage. Science 334, 917-918 (2011).

52. Miller, J. R. Applied physics. Valuing reversible energy storage. Science 335, 1312-1313 (2012)

53. Ferris, A., Garbarino, S., Guay, D. \& Pech, D. 3D RuO 2 microsupercapacitors with remarkable areal energy. Adv. Mater. 27, 6625-6629 (2015).

54. Pech, D. et al. Ultrahigh-power micrometre-sized supercapacitors based on onion-like carbon. Nat. Nanotechnol. 5, 651-654 (2010).

55. Ko, Y. et al. Flexible supercapacitor electrodes based on real metal-like cellulose papers. Nat. Commun. 8, 536 (2017).

56. Zhdanov, V. P., Schwind, M., Zorić, I. \& Kasemo, B. Overheating and undercooling during melting and crystallization of metal nanoparticles. Phys. E Low-Dimens. Syst Nanostruct. 42, 1990-1994 (2010)

57. Coventry, M. D., Allain, J. P. \& Ruzic, D. N. Temperature dependence of liquid $\mathrm{Sn}$ sputtering by low-energy $\mathrm{He}^{+}$and $\mathrm{D}^{+}$bombardment. J. Nucl. Mater. 335, 115-120 (2004).

58. Srinivasan, V. A Critical Assessment of In-flight Particle State During Plasma Spraying of YSZ and its Implications on Coating Properties and Process Reliability. PhD Thesis, State University of New York at Stony Brook (2007).

59. Matejicek, J., Sampath, S., Brand, P. C. \& Prask, H. J. Quenching, thermal and residual stress in plasma sprayed deposits: NiCrAlY and YSZ coatings. Acta Mater. 47, 607-617 (1999).

60. Chen, G. Z. Supercapacitor and supercapattery as emerging electrochemical energy stores. Int. Mater. Rev. 62, 173-202 (2017).

61. Wu, Z., Li, L., Yan, J. M. \& Zhang, X. B. Materials design and system construction for conventional and new-concept supercapacitors. Adv. Sci. 4 1600382 (2017)

62. Stoller, M. D., Park, S., Zhu, Y., An, J. \& Ruoff, R. S. Graphene-based ultracapacitors. Nano Lett. 8, 3498-3502 (2008).

63. El-Kady, M. F. \& Kaner, R. B. Scalable fabrication of high-power graphene micro-supercapacitors for flexible and on-chip energy storage. Nat. Commun. 4, 1475 (2013).

64. Nakanishi, H. \& Grzybowski, B. A. Supercapacitors based on metal electrodes prepared from nanoparticle mixtures at room temperature. J. Phys. Chem. Lett. 1, 1428-1431 (2010).

65. Yoo, J. J. et al. Ultrathin planar graphene supercapacitors. Nano Lett. 11, 1423-1427 (2011).

66. Pech, D. et al. Elaboration of a microstructured inkjet-printed carbon electrochemical capacitor. J. Power Sources 195, 1266-1269 (2010).

67. Peng, Z. et al. Flexible boron-doped laser induced graphene microsupercapacitors. ACS Nano 9, 5868-5875 (2015). 
68. Wang, W. et al. Hydrous ruthenium oxide nanoparticles anchored to graphene and carbon nanotube hybrid foam for supercapacitors. Sci. Rep. 4, 4452 (2014).

69. Dinh, T. M. et al. Hydrous $\mathrm{RuO}_{2}$ /carbon nanowalls hierarchical structures for all-solid-state ultrahigh-energy-density micro-supercapacitors. Nano Energy 10, 288-294 (2014).

70. Yoshida, A., Nonaka, S., Aoki, I. \& Nishino, A. Electric double-layer capacitors with sheet-type polarizable electrodes and application of the capacitors. $J$. Power Sources 60, 213-218 (1996).

71. Askari, S. et al. Silicon-based quantum dots: synthesis, surface and composition tuning with atmospheric pressure plasmas. J. Phys. D. Appl. Phys. 48, 314002 (2015).

72. Cullen, P. J. et al. Translation of plasma technology from the lab to the food industry. Plasma Process. Polym. 15, 1700085 (2018).

\section{Acknowledgements}

This work was supported by the Marie Curie Initial Training Network (RAPID-ITN, award n.606889) and by EPSRC (awards n.EP/K022237/1, n.EP/M024938/1 and n. EP/ M015211/1). We would like to acknowledge Beatrix Biskup (Ruhr-university Bochum, Germany) for taking the photos in Fig. 1.

\section{Author contribution}

A.H. designed, performed experiments, collected data, analyzed and wrote the manuscript; S.A. contributed with TEM analysis; A.M., S.R. \& J.D. contributed with the electrochemical measurements; S.C. \& P.P. contributed to the analysis of the electrochemical results; D.M. V.S. \& P.M. contributed to the initial research idea; D.M. supervised the project and co-wrote/reviewed the paper. All authors contributed to the write-up and provided feedback to the final version of the manuscript.

\section{Additional information}

Supplementary Information accompanies this paper at https://doi.org/10.1038/s41467019-08661-9.

Competing interests: The authors declare no competing interests

Reprints and permission information is available online at http://npg.nature.com/ reprintsandpermissions/

Journal peer review information: Nature Communications thanks Bong Gill Choi and the other anonymous reviewers for their contribution to the peer review of this work. Peer reviewer reports are available.

Publisher's note: Springer Nature remains neutral with regard to jurisdictional claims in published maps and institutional affiliations.

(c) (i) Open Access This article is licensed under a Creative Commons Attribution 4.0 International License, which permits use, sharing, adaptation, distribution and reproduction in any medium or format, as long as you give appropriate credit to the original author(s) and the source, provide a link to the Creative Commons license, and indicate if changes were made. The images or other third party material in this article are included in the article's Creative Commons license, unless indicated otherwise in a credit line to the material. If material is not included in the article's Creative Commons license and your intended use is not permitted by statutory regulation or exceeds the permitted use, you will need to obtain permission directly from the copyright holder. To view a copy of this license, visit http://creativecommons.org/ licenses/by/4.0/.

(C) The Author(s) 2019 